\title{
Mechanical properties of granular materials: A variational approach to grain-scale simulations
}

\author{
R. Holtzman ${ }^{1}, *$ D. B. Silin ${ }^{1,2}$ T. W. Patzek ${ }^{1,2}$ \\ ${ }^{1}$ Department of Civil and Environmental Engineering, University of California, Berkeley, CA \\ 94720, USA \\ ${ }^{2}$ Earth Sciences Division, Lawrence Berkeley National Laboratory, Berkeley, CA 94720, USA
}

\begin{abstract}
SUMMARY
The mechanical properties of cohesionless granular materials are evaluated from grain-scale simulations. A three-dimensional pack of spherical grains is loaded by incremental displacements of its boundaries. The deformation is described as a sequence of equilibrium configurations. Each configuration is characterized by a minimum of the total potential energy. This minimum is computed using a modification of the conjugate gradient algorithm.

Our simulations capture the nonlinear, path-dependent behavior of granular materials observed in experiments. Micromechanical analysis provides valuable insight into phenomena such as hysteresis, strain hardening and stress-induced anisotropy. Estimates of the effective bulk modulus, obtained with no adjustment of material parameters, are in agreement with published experimental data. The model is applied to evaluate the effects of hydrate dissociation in marine sediments. Weakening of the sediment is quantified as a reduction in the effective elastic moduli.
\end{abstract}

Key Words: granular material; quasi-static deformation; grain-scale simulations; variational approach; micromechanics; hydrate dissociation

\section{INTRODUCTION}

The mechanical properties of cohesionless granular materials are important in many applications, making them the subject of intensive research [1]. When a granular material undergoes deformation, its bulk response is determined by the interactions among discrete grains. This property of granular materials makes grain-scale numerical simulations a valuable tool [2-5]. For instance, such simulations provide contact forces in three-dimensions (3D) which are currently unavailable from experiments [5], and enable insight into the mechanical response of granular matter [6].

Interactions between grains are modeled by a contact law, relating the loads at the grain contacts to their relative displacements and deformations. For elastic deformations, the force 
component acting normal to the contact interface can be calculated by the Hertzian model [7], which has been verified by experiments [8]. To evaluate loads related to intergranular friction such as tangential force and torsional couple, the model by Mindlin and Deresiewicz [9] (M-D) is considered adequate [10]. Since it is cumbersome to implement M-D theory for multiple contacts, simplified versions are used for computations, e.g. [10]. However, simulations show that the normal components of the contact forces dominate many aspects of the bulk response [11-14]. Thus, essential aspects of the mechanics of granular materials can be captured by using a "frictionless" contact law, accounting for the normal contact forces only [15].

Deformation can be described by a quasi-static model, as a sequence of equilibrium configurations, corresponding to incremental changes of the boundary conditions. These configurations are found by using a discrete model. Most grain-scale simulations are based on the Discrete Element Method (DEM) [16]. In DEM, interactions between the grains are treated as a dynamic process, accounting for their inertia. The equilibrium configurations are found by numerical integration over time. The tight constraints imposed on the time step used in the integration [17], make DEM simulations time consuming. To accelerate computations and damp grain oscillations, material properties such as the density of the grains and their contact stiffness are often adjusted $[13,18]$ to unrealistic values.

In this paper, we present a technique which does not require such adjustments. The input parameters in our model are the sizes, densities and elastic moduli of the grains, obtained from published experiments. We use a quasi-static model, where each load increment is followed by a static equilibrium. The contact forces are determined by the Hertzian model, assuming frictionless contacts. Thus, all forces can be obtained as gradients of a potential field. Employing a variational approach, we seek for the equilibrium configurations by minimizing the total potential energy of the system. A similar variational approach was employed in other discrete models $[19,20]$.

Three-dimensional micromechanical analysis is used to demonstrate mechanisms leading to phenomena such as hysteresis and stress-induced anisotropy. Macroscopically, our simulations capture the nonlinear, path-dependent response observed in experiments. With no adjustment of material parameters, our estimates of the effective bulk modulus are in agreement with published experimental data. However, the shear modulus is underestimated; this is attributed to the frictionless contact model employed. Elsewhere [21], we extend our variational approach to account for intergranular friction. This extension produces more accurate predictions of the moduli.

Our model is applied to evaluate the mechanical response to dissociation of gas-hydrates in marine sediments. Hydrate dissociation caused, for instance, by hot fluids produced from deep formations, can destabilize the ocean floor and lead to landslides [22], risking nearby structures such as offshore platforms. The mechanical properties and structure of hydratebearing sediments (HBS) are not well-known, due to difficulties in in-situ sampling and creation of representative artificial samples [23]. We quantify the degradation of strength in an HBS sample by a reduction in elastic moduli.

The outline of this paper is as follows. Section 2 describes our model and the simulations methodology. Grain-scale and macroscopic observations from our simulations are presented in Section 3. In Section 4, we quantify the effects of hydrate dissociation in marine sediments. We discuss our results in Section 5 . 


\section{SIMULATING DEFORMATION OF GRANULAR MATERIALS}

We simulate loading of cohesionless grains packed in a rectangular container. Load is applied by incremental displacements of the container walls, allowing the grains to reach static equilibrium. Each equilibrium configuration is computed by minimizing the total potential energy of the system. The initial conditions are the positions of the grains, prior to the simulated test. The positions and orientations of the container walls determine the boundary conditions, from which the strains are evaluated. By applying uniform or different normal strains in three principal directions, we simulate an isotopic or a polyaxial test. A uniaxial test is simulated by loading in a single direction.

\subsection{Grain Pack Model}

We model granular matter as a heterogeneous, irregular ("random") 3D packing of spherical grains, see Figure 1. Each grain is homogenous and isotropic. To model heterogenous materials such as clastic sediments, we assign the grain sizes and elastic moduli from a given distribution. The deformations are assumed to be small and localized near the contacts, allowing for the shape of a deformed grain to be approximated by a sphere.
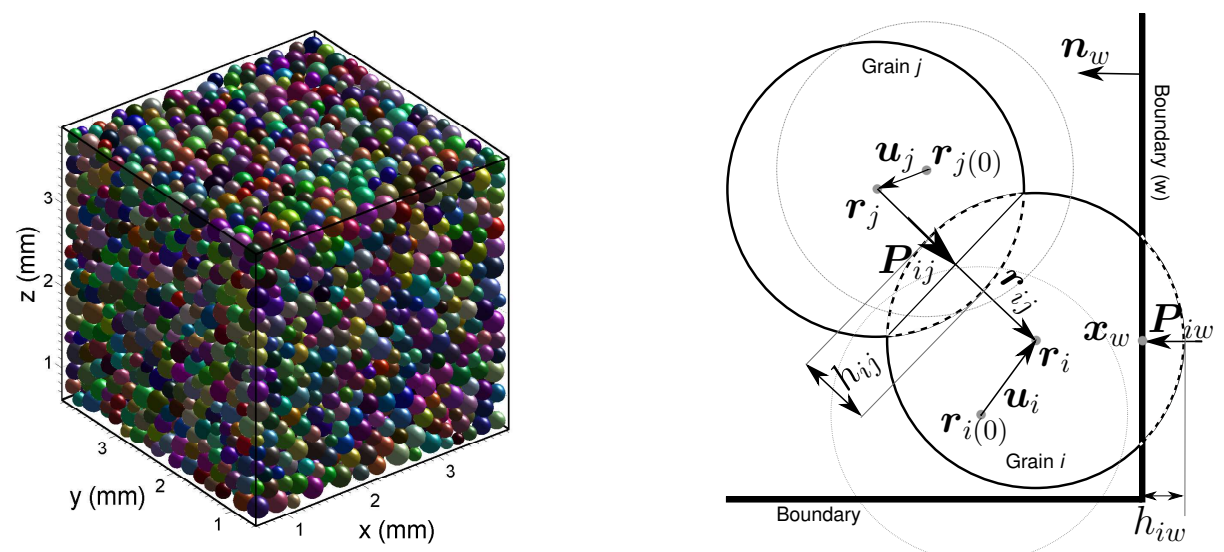

Figure 1. Left: a typical pack with 5036 grains used in the numerical simulations. Right: schematic description of the contact geometry. The initial configuration of the grains is marked with grey dotted lines. The dashed lines represent grain overlap caused by deformations.

In a fixed coordinate system, the geometry of each grain is fully described by the coordinates of its center and its radius. This is since the orientations of the grains are of no importance under the assumption of frictionless contacts. Thus, for a 3D pack of $N$ grains, each configuration has $3 N$ degrees of freedom, i.e. the grain centers coordinates. Labeling all grains with a single index $i=1,2, \ldots, N$, we denote the radius-vector of the center of grain $i$ by $\boldsymbol{r}_{i}$. Given a reference configuration in equilibrium, we perturb the boundary conditions, forcing the grains to deform and rearrange to a new equilibrium configuration ("current"). Let $\boldsymbol{u}_{i}$ denote the displacement of grain $i: \boldsymbol{u}_{i}=\boldsymbol{r}_{i}-\boldsymbol{r}_{i(0)}$, where subscript (0) denotes the reference configuration, see Figure 1 . The radius of grain $i$ is $R_{i}$, its mass is $m_{i}$, and the Young's modulus and Poisson's ratio of its material are $E_{i}$ and $\nu_{i}$, respectively. Subscript $w$ denotes a boundary 
wall.

We hypothesize here that in a tight packing, the macroscopic stress is mostly affected by the normal component of the contact forces [11-14]. This assumption is based on the argument that the size of the asperities that resist lateral or angular displacements is much smaller than the size of the grains themselves. Thus, we only account for the normal force components.

\subsection{Contact Model: Force-Displacement Relation}

According to Hertzian contact theory, the magnitude of the compressive normal force acting at the contact between a pair of grains $i$ and $j$ is

$$
\left\|\boldsymbol{P}_{i j}\right\|=\frac{4}{3} E_{i j}^{*}\left(R_{i j}^{*}\right)^{1 / 2}\left(h_{i j}\right)^{3 / 2}
$$

where $\|\boldsymbol{a}\|=(\boldsymbol{a} \cdot \boldsymbol{a})^{1 / 2}$ is the magnitude of a vector $\boldsymbol{a}$. Parameters $R_{i j}^{*}=\left(\frac{1}{R_{i}}+\frac{1}{R_{j}}\right)^{-1}$ and $E^{*}=\left[\frac{\left(1-\nu_{i}^{2}\right)}{E_{i}}+\frac{\left(1-\nu_{j}^{2}\right)}{E_{j}}\right]^{-1}$ are the effective radius and elastic coefficient associated with that contact, respectively. Eq. (1) is applicable to a grain-boundary contact, by modeling the latter as a grain of infinite radius. The contact deformation is measured by the overlap $h_{i j} \geq 0$,

$$
\begin{array}{ll}
h_{i j}=R_{i}+R_{j}-\left\|\boldsymbol{r}_{i j}\right\| & \text { for grain-grain contact } \\
h_{i w}=R_{i}-\left(\boldsymbol{r}_{i}-\boldsymbol{x}_{w}\right) \cdot \boldsymbol{n}_{w} & \text { for grain-boundary contact }
\end{array}
$$

where $\boldsymbol{r}_{i j}=\boldsymbol{r}_{i}-\boldsymbol{r}_{j}$, the initial contact point with the wall $w$ is $\boldsymbol{x}_{w}$, and $\boldsymbol{n}_{w}$ is an inward unit normal to the wall, see Figure 1. As long as the orientation $\boldsymbol{n}_{w}$ is held fixed, $\boldsymbol{x}_{w}$ can be chosen arbitrarily on the boundary $w$. The force acting on grain $i$ at the contact with another grain $j$ or a wall $w$ is directed along $\boldsymbol{r}_{i j}$ or $\boldsymbol{n}_{w}$, respectively. No moment relative to the center of the grain is developed.

\subsection{Obtaining Equilibrium Configurations}

At equilibrium, the balance of forces for each grain yields three scalar equations, a total of $3 N$ equations for $N$ grains. The unknowns, grain displacements, can be written as a column vector $\boldsymbol{\theta}=\left[\boldsymbol{u}_{1} \ldots \boldsymbol{u}_{N}\right]^{T}$. Here $\boldsymbol{u}_{i}$ is a row vector, and superscript $T$ is the transpose. The exponent of $3 / 2$ in Eq. (1) makes the system of equations nonlinear.

We seek an equilibrium configuration by employing a variational formulation. Namely, an equilibrium configuration is characterized by a set of displacements $\boldsymbol{\theta}$ corresponding to a minimum of the total potential energy of the system. This minimum is found numerically using a modification of the conjugate gradient algorithm [24], see Appendix. The total energy is a function of the deformation (strain energy) and the gravitational energy of the grains. The elastic strain energy of a pair of grains in contact is equal to the sum of work done on each grain to deform it. This work is the dot product of the force with the displacement increment, integrated over the total displacement. Thus, the strain energy $U_{i j}$ stored in the deformed contact region between grains $i$ and $j$ is (cf. Eq. (9.15) in [25]): 


$$
U_{i j}\left(\boldsymbol{u}_{i}, \boldsymbol{u}_{j}\right)=\frac{8 E_{i j}^{*}\left(R_{i j}^{*}\right)^{1 / 2}}{15}\left(h_{i j}\right)^{5 / 2}
$$

The total potential energy of the system is the sum of the strain energy of all contacts and the gravitational potentials of all grains

$$
\Pi(\boldsymbol{\theta})=\sum_{i=1}^{N}\left\{\frac{1}{2} \sum_{j=1}^{N_{g}^{i}} U_{i j}+\sum_{w=1}^{N_{b}^{i}} U_{i w}+m_{i} g\left(\boldsymbol{r}_{i} \cdot \hat{\boldsymbol{e}}_{z}-z^{*}\right)\right\}
$$

Here $z^{*}$ is an arbitrary fixed reference elevation, $\hat{\boldsymbol{e}}_{z}$ is a unit vector pointing opposite to the direction of gravity, and $g$ is the gravity acceleration. By $N_{g}^{i}$ and $N_{b}^{i}$ we denote the number of contacts of grain $i$ with other grains and boundaries, respectively. The coordination number of grain $i$ is $N_{g}^{i}+N_{b}^{i}$. Note that the set of contacts for each grain varies with the deformation of the pack, introducing additional nonlinearity.

The gradient of $\Pi$ with respect to $\boldsymbol{\theta}$ is a column vector $\nabla_{\boldsymbol{\theta}} \Pi=-\left[\boldsymbol{F}_{1} \ldots \boldsymbol{F}_{N}\right]^{T}$, where the row vector $\boldsymbol{F}_{i}$ is the sum of forces on grain $i$,

$$
\boldsymbol{F}_{i}=\sum_{j=1}^{N_{g}^{i}} \boldsymbol{P}_{i j}+\sum_{w=1}^{N_{b}^{i}} \boldsymbol{P}_{i w}-m_{i} g \hat{\boldsymbol{e}}_{z}
$$

Thus, the vanishing gradient of $\Pi$ is equivalent to the balance of forces.

\subsection{Initial Grain Pack Generation}

To simulate deformation of a grain pack, a sufficiently dense initial configuration is required $[26,27]$. Such an initial configuration could be obtained from a physical sample, using advanced imaging $[28,29]$. Alternatively, an initial configuration could be generated numerically using either "constructive" or "dynamic" algorithms. Constructive algorithms are based on geometry alone and thus require relatively small computing time; however, the current state-of-the-art constructive algorithms produce 3D arrangements with low coordination numbers, gaps, or anisotropic structure [27]. In dynamic algorithms, a loose packing is created by placing a relatively small number of grains in a bounded domain. Then, its density is increased by either expanding the grains or moving the boundaries closer together. A static equilibrium configuration is found by simulating intergranular interactions, e.g. using DEM. The large number of collisions and grain rearrangements makes such a procedure time-consuming [27].

Instead, we use a quasi-static algorithm. Here, we start by selecting a portion of a pack generated by DEM [30], bounding it by a rectangular domain. This packing is loose, with many grains supported by less than 4 contacts, rendering them mechanically unstable. To obtain a denser packing we first expand these unstable grains, until their coordination numbers is at least 4 . Then, we shrink the pack by equal displacements of all boundaries, and apply our algorithm to obtain an equilibrium configuration. This process is repeated until practically all grains have coordination number of 4 or more, and appreciable contact forces develop.

\subsection{Evaluating Macroscopic Parameters}

2.5.1. Stress and Strain. There are several methods for transforming between discrete, grainscale parameters and macroscopic, continuum stress and strain (e.g. [31,32]). We consider the 
pack to be a representative volume of a larger medium, and compute the average stress and strain within this volume as described below.

The external normal forces applied on the boundaries are calculated from the sum of the contact forces on the container walls. The average normal stresses are evaluated by dividing these external forces by the area of the respective walls. To confirm the validity of our stress evaluation procedure, we also calculate the averaged Cauchy stress, taking into account the entire set of contact forces within the sample volume [31]. This calculation yields values similar to those obtained from our procedure.

To estimate numerically the macroscopic strains $\boldsymbol{\epsilon}=\left[\nabla_{\boldsymbol{x}} \boldsymbol{u}+\left(\nabla_{\boldsymbol{x}} \boldsymbol{u}\right)^{T}\right] / 2$, we replace the derivatives with finite differences. In the last equation, $\boldsymbol{x}$ is a radius-vector to an infinitesimal volume, and $\boldsymbol{u}$ is its displacement [25]. For a rectangular domain, the normal strain in the $l$ direction $(l=1,2,3)$ reduces to $\left[L_{l}-L_{l(0)}\right] / L_{l(0)}$, where $L_{l}$ and $L_{l(0)}$ are the current and the reference (undeformed) length of the domain in that direction, respectively. In our simulations, the strains determine the boundary conditions, enforced by displacing the container walls.

2.5.2. Elastic Moduli. To describe the mechanical properties as they evolve with the deformation, we discretize the load path, and assign a set of constant effective elastic moduli for each loading interval. The moduli for each interval are evaluated by fitting the stress-strain results with Hooke's law. Hooke's law for a homogenous, isotropic body is $\boldsymbol{\sigma}=\lambda \operatorname{tr}(\boldsymbol{\epsilon}) \hat{\boldsymbol{I}}+2 G \boldsymbol{\epsilon}$, where $\boldsymbol{\sigma}$ is the stress tensor, $\operatorname{tr}(\boldsymbol{\epsilon})$ is the trace of $\boldsymbol{\epsilon}$, and $\hat{\boldsymbol{I}}$ is a unit second order tensor. The moduli $\lambda$ and $G$ are Lame's constant and the shear modulus, respectively. Other elastic moduli can be evaluated from $\lambda$ and $G$ [25].

The moduli evaluated using the procedure above are the bulk-averaged, effective moduli, corresponding to an effective homogeneous and isotropic elastic medium. Because the moduli depend on the loads, stress-induced anisotropy can develop when loads in different directions are significantly different [33]. This phenomenon was observed in our simulations, see Section 3.3. To verify whether a grain pack is isotropic, we simulate a polyaxial test and compare the moduli for each pair of principal directions. If they are similar within a given tolerance, we consider the response isotropic. We minimize stress-induced anisotropy by applying relatively isotropic strains, evaluating the moduli at different loads by a uniaxial test. Macroscopically isotropic systems may exhibit local anisotropy, if the number of grains within the selected volume is too small [15]. To avoid such scale-related anisotropy, we use packs with sides not smaller than $\sim 15$ grain diameters. This length scale depends on the properties of the grains and their spatial arrangement, and was determined by trial and error. As a result of using the above measures, the estimates of the moduli in different directions are practically identical.

\section{SIMULATIONS}

\subsection{Model Parameters}

To verify our model, we compare our simulation results with published experiments. In particular, we compare our results to experiments on glass beads, since they are relatively spherical and smooth. Thus, the elastic moduli of the grains were assigned mean values of $\bar{E}=70 \mathrm{GPa}$ for Young's modulus and $\bar{\nu}=0.2$ for Poisson's ratio (corresponding to bulk and shear modulus of $\bar{K}=38.9 \mathrm{GPa}$ and $\bar{G}=29.2 \mathrm{GPa}$, respectively), similar to glass beads 

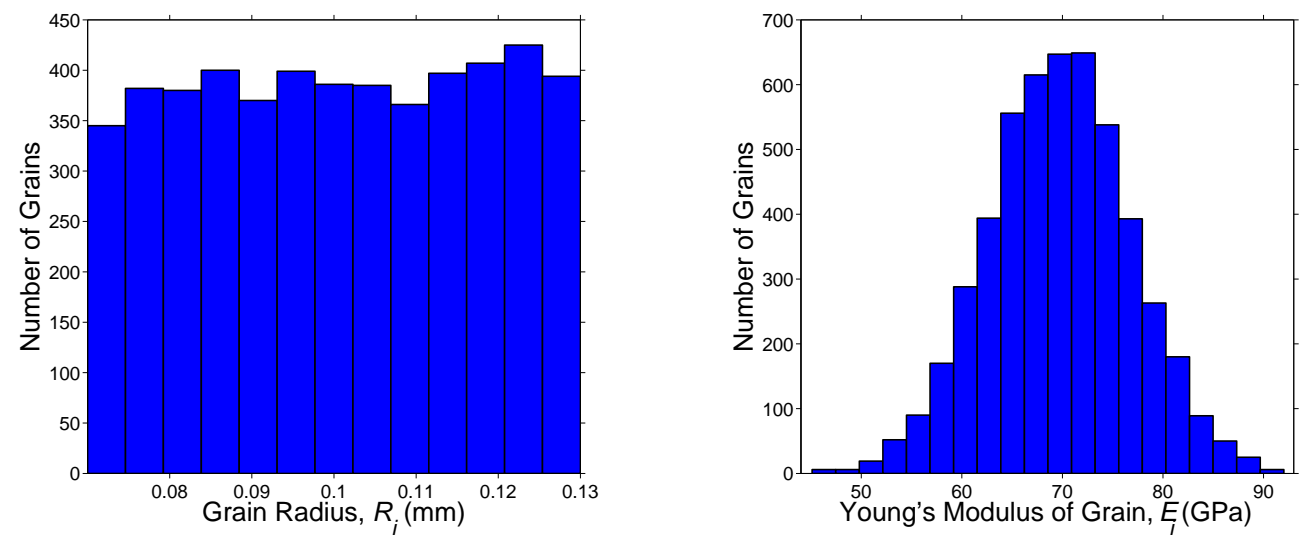

Figure 2. The distribution of the grain sizes (left) and Young's modulus values (right) for pack G5036, representing a sample made of 5036 glass beads.

[34]. In other simulations, quartz sand was modeled by assigning $\bar{E}=100 \mathrm{GPa}$ and $\bar{\nu}=0.15$ $(\bar{K}=47.6 \mathrm{GPa}$ and $\bar{G}=43.5 \mathrm{GPa})[35,36]$. The elastic moduli were normally distributed with a standard deviation of $10 \%$ of the mean. The radii of the grains used to generate the initial pack were distributed uniformly between 0.07-0.13 mm. Since the initial pack generation procedure involves expansion of the unstable grains, the grain radii slightly deviate from this distribution. The density of the glass and quartz grains was taken to be 2.42 and $2.65 \mathrm{~g} / \mathrm{cm}^{3}$ [35]. The number of grains used was 5036 and 2654 for the glass beads and quartz sand, denoted by G5036 and Q2654, respectively. The distribution of the grain sizes and Young's modulus values for G5036 are plotted in Figure 2.

\subsection{Macro-mechanical Analysis}

3.2.1. Stress-Strain. To capture hysteresis and stress-induced anisotropy, we simulated a loading-unloading cycle in a polyaxial test on sample Q2654. The porosity and mean coordination number varied as 35.8-30.5\% and 7.23-8.26, accordingly, with stresses of $\sim 60$ $300 \mathrm{MPa}$. The stress-strain curves calculated for Q2654 are plotted in Figure 3. The abrupt change in the slopes is associated with variations in the complex network of contacts ("fabric") and contact forces. Some of these microstructural variations are irreversible, contributing to the hysteretic response observed in experiments [37]. This hysteresis is evident in Figure 3 as different loading and unloading curves.

3.2.2. Elastic Moduli. To verify our model against experiments on glass beads, we simulated isotropic compression of sample G5036, evaluating the macroscopic elastic moduli for different loads by an incremental uniaxial loading. In these simulations, the porosity and mean coordination number varied as 37.4-35.1\% and 6.24-7.15, accordingly, with stresses of $\sim 1-35$ $\mathrm{MPa}$. Our moduli estimates are plotted in Figure $4 v s$. the confining stress $\sigma_{c}$, defined by the mean of the lateral stresses perpendicular to the principal loading direction. Figure 4 also shows results of acoustic experiments, simulations and analytical solutions by [34,35,38], conducted using packs of glass beads with micro-properties similar to those used in our simulations. The 


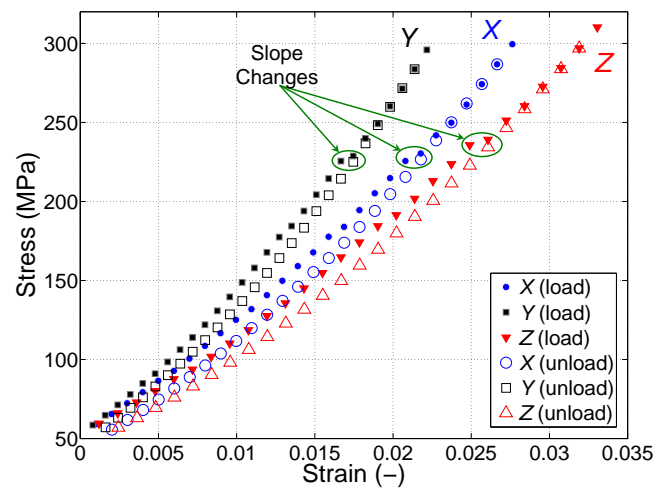

Figure 3. Stress-strain curves obtained from simulations of a polyaxial loading-unloading cycle on sample Q2654. Hysteresis is evident as different loading and unloading curves. This hysteresis is related to the abrupt change in the slopes (encircled).

DEM and EMT calculations in [34] employed a contact law which prohibits sliding of grains, i.e. assigning infinitely large intergranular friction.

Our bulk modulus $(K)$ estimates are in good agreement with the published experimental data, with some values slightly lower than the data. In addition, except at $\sigma_{c}<2 \mathrm{MPa}$, our evaluated shear modulus $(G)$ growth agrees with the experimental data, showing faster growth rate than the power law $\sigma_{c}{ }^{1 / 3}$ predicted by the EMT [39]. The discrepancy between the EMT predictions and experimental data is discussed in [34,40,41].

At the same time, our shear modulus strongly underestimates the experimental data. In particular, at low stresses, the evaluated shear modulus drops sharply with the stress. Loss of shear rigidity is observed as the mean coordination number approaches 6 and the porosity exceeds $\sim 37 \%$. However, loss of rigidity is expected to occur at lower packing densities, as intergranular friction strengthens the pack [42]. We associate the discrepancies between our predictions and the data with the frictionless contact assumption, because the shear modulus greatly depends on the intergranular shear [42].

\subsection{Micromechanical Analysis}

Relating grain-scale parameters to macroscopic, continuum parameters can improve our understanding of the complex behavior of granular materials [14]. The effect of the spatial distribution of grain-scale parameters cannot be captured by averaged, macroscopic quantities. For example, by tracking the contact forces larger than a certain threshold, we observe force chains, as most of the load is carried by relatively few grains. This can lead to fracturing and failure of the material, which cannot be predicted from averaged quantities such as stress. Force chains have been observed in experiments using photoelastic disks (e.g. [43]) and in DEM simulations (e.g. [3, 6, 12, 15, 42]).

Correspondence between abrupt change in the slopes of the stress-strain curves and substantial variations in the contact network and the force chains was noticed in our simulations. A similar correlation was observed between deviator stress drops and microstructural variations [3]. Some of these variations are irreversible, contributing to 

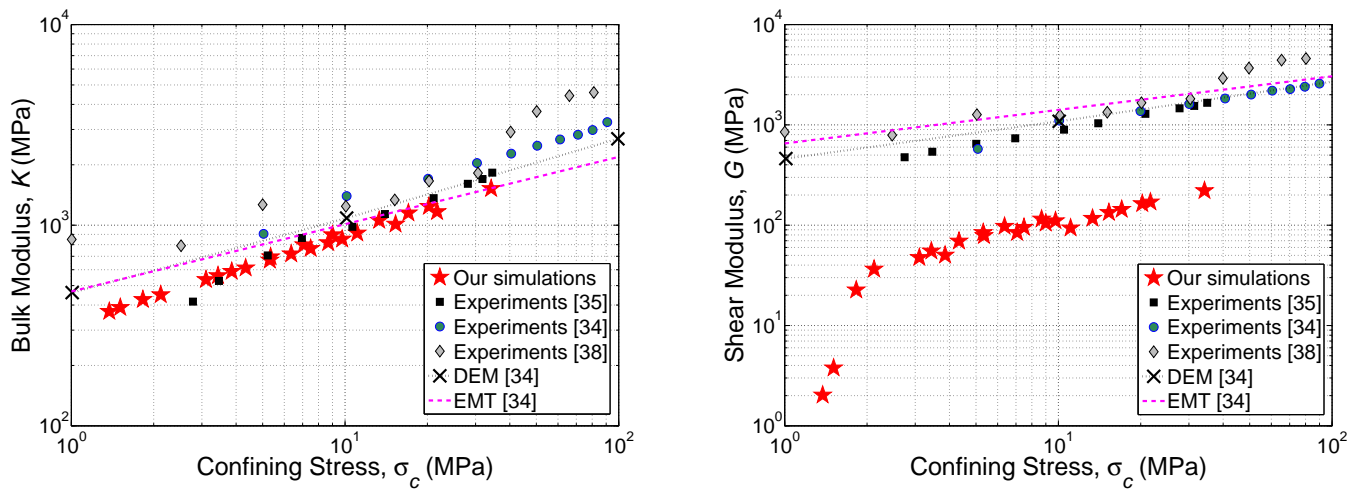

Figure 4. The effective bulk (left) and shear (right) modulus vs. the confining stress $\sigma_{c}$. The evaluated moduli from simulations on sample G5036 are compared with published results of acoustic experiments in glass beads $[34,35,38]$, in addition to DEM simulations and effective medium theory (EMT) calculations by [34]. The published data was digitized from Figure 1 in [34].

the hysteretic bulk response. Tracking hysteresis via grain-scale parameters can be done quantitatively using the fabric tensor [37]. Qualitatively, we observe that these abrupt changes were correlated to relatively large displacements experienced by several grains. These displacements are possible at particular combinations of contact forces and geometry, as grains are "pushed" through constrictions. Following such events, the local set of contacts and the shapes of these constrictions are altered significantly, so that a reverse-perturbation of the boundary conditions cannot restore the original configuration. In loading, these rearrangements create an overall stiffer pack, which may be interpreted as strain hardening.

Stress-induced anisotropy was observed in simulations of highly anisotropic loading on sample Q2654. To investigate the mechanisms leading to such anisotropy, we analyzed the directions of the contact forces which were larger than the mean. Under isotropic loads these directions were uniformly distributed. In contrast, under anisotropic loads corresponding to anisotropic moduli, these forces were preferentially aligned with the direction of the maximum external load. Similar observations were made from experiments [43] and simulations [12].

\section{IMPACT OF HYDRATE DISSOCIATION IN MARINE SEDIMENTS}

\subsection{Modeling Hydrate Dissociation}

We apply our model to quantify the mechanical response to hydrate dissociation in HBS. Gashydrates are solid materials formed under a range of high pressures and low temperatures. Dissociation converts them into liquid water and free gas. The volumetric expansion of gas can lead to an appreciable increase in pore pressure [22], reducing the effective stress. Thus, dissociation impacts the mechanical properties of HBS by reducing both the solid fraction of the sediment and the effective stress.

The pressure increase varies with factors such as dissociation rate, sediment permeability, and initial pore pressure [22]. Predicting the excess pressure as a function of dissociation is 
outside the scope of this paper. Thus, we consider a number of dissociation scenarios. A range of dissociation amounts and excess pressures, taken from other models [22], is used as input parameters in our simulations.

The distribution of hydrates within the pore space depends on the formation mechanism, among other factors, and a wide variety of distribution models exists [44]. To model interactions between the host sediment and the hydrate, we consider methane-hydrate formed preferentially in the larger pores, rather than in the pore-throats. This type of hydrate distribution can occur if small amounts of hydrates are slowly introduced in the aqueous phase [23]. Such a distribution makes hydrates a part of the solid skeleton, i.e. load-bearing solid particles.

We quantify the amount of dissociation by the decrease in hydrate saturation from $S$ to $S+\Delta S$, where $\Delta S<0$. The hydrate saturation is defined by $S=V^{h} / V$, where $V^{h}$ is the volume occupied by hydrate and $V$ is the total pore volume, including the hydrate. We model the reduction in solid fraction due to dissociation by shrinking the hydrate grains, assuming all grains experience a similar volumetric strain. The impact of the excess pore pressure, $p_{e x}$, is modeled at both the grain and the sample level. We assume uniform pore pressure within the sample. Since the contact area is much smaller than the surface area of the grains, we isotropically compress the grains, neglecting the net force applied by the fluid pressure. The volume of grain $i, V_{i}$, is changed by $\Delta V_{i}<0$. For small changes in volume, $\Delta V_{i} / V_{i}$ is approximately equal to the volumetric strain, and can be determined by $-p_{e x}=K_{i} \Delta V_{i} / V_{i}$. Here $K_{i}$ is the bulk modulus of grain $i$. At the sample scale, the reduction in effective stress is modeled by applying a tensile macroscopic strain $\epsilon_{k k}>0$, expanding the sample isotropically. This strain is determined from the poroelastic constitutive equation $\alpha_{b} p_{e x}=K \epsilon_{k k}$, where $\alpha_{b}$ is the Biot-Willis coefficient [45].

\subsection{Elastic Moduli Following Hydrate Dissociation}

The effect of dissociation in HBS is quantified via the evolution of the elastic moduli. The initial state of an HBS sample is modeled as a dense arrangement of spherical grains, where some of the smallest grains are methane-hydrate. Hydrates are assigned with elastic moduli of $E_{i}=6.6 \mathrm{GPa}$ and $\nu_{i}=0.32\left(K_{i}=6.1 \mathrm{GPa}\right.$ and $\left.G_{i}=2.5 \mathrm{GPa}\right)$, and density of $0.9 \mathrm{~g} / \mathrm{cm}^{3}$ [46]. To model quartz sand as the host sediment, the other grains were assigned properties of quartz, see Section 3.1. A typical value for sand of $\alpha_{b}=0.8$ was used [47]. The initial porosity and hydrate saturation was $\phi \approx 45 \%$ and $S=0.215$. The effective stress for this initial packing, determined from the contact forces on the walls (see Section 3.2.1), was $\sim 21 \mathrm{MPa}$. The initial pore pressure may be larger than hydrostatic pressure if the sediment is confined by a lowpermeability layer. The resulting total stress, which is considered fixed in our simulations, can exceed the lithostatic stress if the sediment carries the weight of a nearby offshore platform.

By varying the excess pressure and the saturation decrement independently, we produce a series of configurations, covering a range of possible scenarios. For each configuration, the elastic moduli are evaluated by simulating a uniaxial test. In Figure 5 (left), these moduli are plotted against the excess pressure, for a fixed saturation decrement, $\Delta S=-0.01$. Conversely, Figure 5 (right) shows the moduli vs. $\Delta S$, for a fixed excess pressure $p_{e x}=1 \mathrm{MPa}$. The initial moduli prior to dissociation are plotted in the left-most parts of both figures.

Weakening of HBS is evident from a reduction in elastic moduli, as the sediment becomes looser and softer. This observation is in qualitative agreement with experiments $[23,44]$ and other numerical simulations [48]. Further dissociation may lead to a significant decrease in 

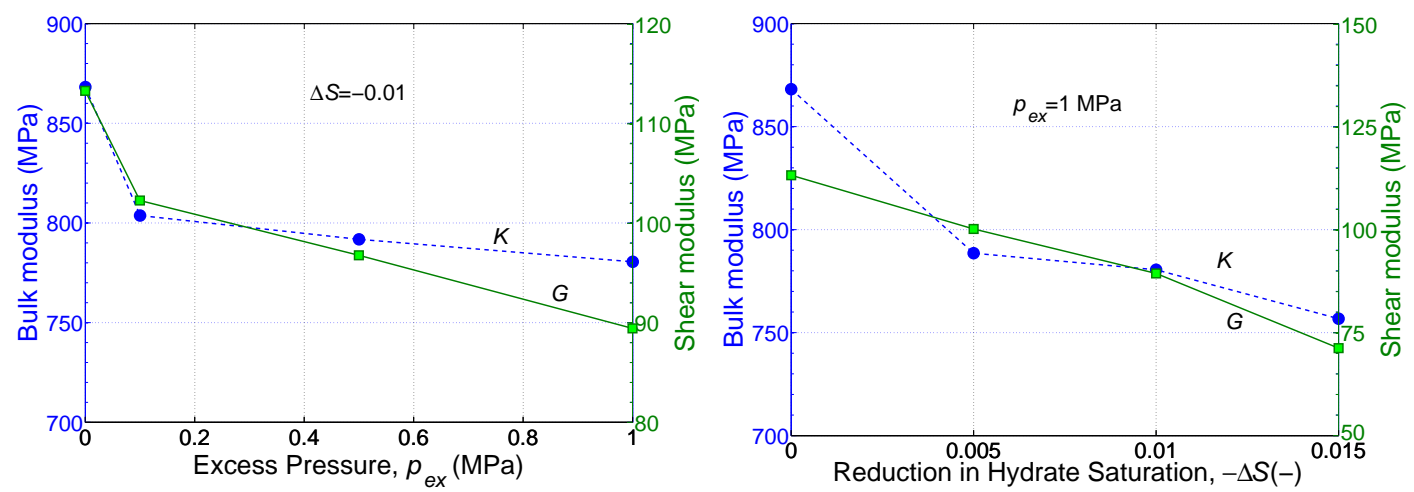

Figure 5. Simulating a reduction in bulk (dashed line) and shear (solid) modulus due to hydrate dissociation. Left: varying the excess pore pressure $p_{e x}$, for a fixed saturation decrement, $\Delta S=-0.01$. Right: varying the saturation decrement for a fixed excess pressures $p_{e x}=1 \mathrm{MPa}$.

the solid support, with a possible loss of shear rigidity. In such a state, the sediment is more susceptible to landslides and subsidence, threatening the safety of adjacent offshore platforms.

\section{DISCUSSION}

A technique for estimating the mechanical properties of cohesionless granular materials from numerical simulations has been presented. We model granular matter as a 3D disordered packing of elastic spherical grains, bounded by a rectangular container. Using a quasi-static model, the pack is loaded by incremental displacements of the container walls. The contact forces are calculated using Hertzian contact theory. Each equilibrium configuration is found by minimizing the total potential energy of the system. A modification of the conjugate gradient algorithm is used to obtain this minimum. This approach results in an efficient computational procedure, which is also used to generate a dense initial arrangement.

By micromechanical analysis we demonstrate mechanisms responsible for hysteresis, strain hardening, and stress-induced anisotropy. Macroscopically, our results capture the nonlinear and path-dependent response observed in experiments. We have verified our physics-based model against published experimental data, using similar grain properties. All material parameters used in our model, i.e. the sizes, densities and elastic moduli of the grains, serve as input parameters which are obtained from published experiments. The good agreement between predicted and measured values of the macroscopic bulk modulus has been achieved with no adjustments of parameters.

These results confirm that the normal contact forces play an important role in determining the overall response, and that grain-scale elasticity is suitable to describe many features of the inelastic response of granular materials. The bulk modulus, for example, mainly depends on the normal contact forces, which are adequately described by the Hertzian theory. Nevertheless, our model underestimates the shear modulus. We attribute this deficiency to the frictionless contact model employed. Extension of our variational approach to account for shear and sliding at the contacts provides more accurate predictions of the moduli [21]. 
We apply our model to quantify the effect of hydrate dissociation in marine sediments. Hydrates are modeled as load-bearing solid particles within the pores. To model dissociation, we reduce the solid fraction by shrinking the hydrate grains. The effect of the related excess pore pressure is modeled by expanding the sample to account for the decrement of effective stress, and compressing the grains. A series of possible scenarios has been simulated, showing degradation in sediment strength as a reduction in the macroscopic elastic moduli. This trend agrees qualitatively with the published results of experiments and numerical simulations. Further dissociation might lead to loss of solid support of the skeleton, causing seafloor landslides and subsidence. To predict such instabilities and their impact on offshore platforms, our model can be used to produce constitutive relationships for large-scale simulations (e.g. [48]). To quantitatively verify our predictions, more experimental data will be required.

\section{ACKNOWLEDGEMENT}

This work was supported by the Assistant Secretary for Fossil Energy, Office of Natural Gas and Petroleum Technology, through the National Energy Technology Laboratory, under the U.S. Department of Energy, Contract \#DE-FC26-05NT42664.

\section{APPENDIX: MINIMIZATION ALGORITHM}

Each equilibrium configuration is found numerically, using a modified conjugate gradient (CG) algorithm. We seek displacements $\boldsymbol{\theta}$ which provide a local minimum of the functional $\Pi$. Iterative updates $\boldsymbol{\theta}^{k+1}=\boldsymbol{\theta}^{k}-\alpha^{k} \boldsymbol{p}^{k}$ are performed until the displacements converge to provide a local minimum of $\Pi$ within a desired accuracy. Superscript $k$ denotes the iteration index. The updates are performed in the so-called search directions $\boldsymbol{p}^{k}=\boldsymbol{R}^{k}-\beta^{k} \boldsymbol{p}^{k-1}$, where $\beta^{k}=-\left(\left\|\boldsymbol{R}^{k}\right\| /\left\|\boldsymbol{R}^{k-1}\right\|\right)^{2}$. The residual vector $\boldsymbol{R}^{k}$ equals the gradient of $\Pi, \boldsymbol{R}^{k}=-\left[\boldsymbol{F}_{1}^{k} \ldots \boldsymbol{F}_{N}^{k}\right]^{T}$.

We use the steepest descent method, finding a value for the scaling coefficient $\alpha^{k}$ which provides a minimum for $\Pi\left(\boldsymbol{\theta}^{k}-\alpha^{k} \boldsymbol{p}^{k}\right)$. To avoid nonphysical artifacts such as grain permutations, the value of $\alpha$ is restricted within each iteration. The iterations are stopped if at least one of the following holds true:

$$
\Pi^{k}-\Pi^{k+1}<\varepsilon_{1} \Pi^{k} \quad \text { or } \quad\left\|\boldsymbol{R}^{k+1}\right\|^{2}<\varepsilon_{2} N\left(\bar{E} \bar{R}^{2}\right)^{2}
$$

where $\bar{R}$ is the mean grain radius. For example, for sample Q2654, $\bar{E}=10^{11} \mathrm{~Pa}$ and $\bar{R}=1.003 \cdot 10^{-4}$ $\mathrm{m}$. The tolerance parameters were chosen to be $\varepsilon_{1}=10^{-10}$ and $\varepsilon_{2}=10^{-3}$. These values provide for each grain a residual force $\boldsymbol{F}_{i}$, which was smaller than the maximum contact force on that grain by at least 3 orders of magnitude. Note that due to inevitable round-off error, the chosen tolerance cannot be arbitrarily small.

For a linear system of the order $3 N$ with a positive-definite matrix, CG is proven to yield an exact solution within $3 N$ iterations [24]. However, the nonlinearities related to the force-displacement relation and variations in the fabric, in addition to accumulated round-off error, may cause loss of convergence, as updating $\boldsymbol{\theta}^{k}$ in the direction of $\boldsymbol{p}^{k}$ may not reduce the value of $\Pi$. To avoid this behavior, we refresh the CG algorithm by performing an update in the direction of the gradient $\boldsymbol{R}^{k}$. The frequency of this operation is determined by trial-and-error. 
1. Bagi K. Granular mechanics Special Issue. International Journal of Solids and Structures 2004; 41(21):5761-5762.

2. Kruggel-Emden H, Simsek E, Rickelt S, Wirtz S, Scherer V. Review and extension of normal force models for the discrete element method. Powder Technology 2007; 171(3):157-173.

3. Peña AA, Lizcano A, Alonso-Marroquin F, Herrmann HJ. Biaxial test simulations using a packing of polygonal particles. International Journal for Numerical and Analytical Methods in Geomechanics 2008; 32(2):143-160.

4. Walsh SDC, Tordesillas A, Peters JF. Development of micromechanical models for granular media. Granular Matter 2007; 9(5):337-352.

5. Cole D, Peters J. A physically based approach to granular media mechanics: grain-scale experiments, initial results and implications to numerical modeling. Granular Matter 2007; 9(5):309-321.

6. Radjai F, Jean M, Moreau JJ, Roux S. Force distributions in dense two-dimensional granular systems. Physical Review Letters 1996; 77(2):274-277.

7. Hertz H. Über die berührung fester elastischer körper (on the contact of elastic solids). Journal reine und angewandte Mathematik 1882; 92:156-171. In German.

8. Johnson KL. Contact Mechanics. Cambridge University Press: Cambridge, MA, 1987.

9. Mindlin RD, Deresiewicz H. Elastic spheres in contact under varying oblique forces. Journal of Applied Mechanics 1953; 20:327-344.

10. Vu-Quoc L, Zhang X. An accurate and efficient tangential force-displacement model for elastic frictional contact in particle-flow simulations. Mechanics of Materials 1999; 31(4):235-269.

11. Rothenburg L, Bathurst RJ. Analytical study of induced anisotropy in idealized granular-materials. Geotechnique 1989; 39(4):601-614.

12. Thornton C, Antony SJ. Quasi-static deformation of particulate media. Philosophical Transactions of the Royal Society A: Mathematical, Physical and Engineering Sciences 1998; 356(1747):2763-2782.

13. Thornton C. Numerical simulations of deviatoric shear deformation of granular media. Geotechnique 2000; 50(1):43-53.

14. Kruyt NP, Antony SJ. Force, relative-displacement, and work networks in granular materials subjected to quasistatic deformation. Physical Review E 2007; 75(5):051308.

15. Goldenhirsch I, Goldenberg C. Stress in dense granular materials. The Physics of Granular Media, Hinrichsen H, Wolf DE (eds.). Wiley-VCH, 2005.

16. Cundall PA, Strack ODL. A discrete numerical model for granular assemblies. Geotechnique 1979; 29:4765 .

17. O'Sullivan C, Bray JD. Selecting a suitable time-step for discrete element simulations that use the central difference time integration approach. Engineering Computations 2004; 21(2-4):278-303.

18. O'Sullivan C, Bray JD, Riemer M. Examination of the response of regularly packed specimens of spherical particles using physical tests and discrete element simulations. Journal of Engineering Mechanics 2004; 130(10):1140-1150.

19. O'Hern CS, Silbert LE, Liu AJ, Nagel SR. Jamming at zero temperature and zero applied stress: The epitome of disorder. Physical Review E 2003; 68(1):011 306.

20. Renouf M, Alart P. Conjugate gradient type algorithms for frictional multi-contact problems: applications to granular materials. Computer Methods in Applied Mechanics and Engineering 2005; 194:2019-2041.

21. Holtzman R, Silin DB, Patzek TW. Deformations of sediments via grain-scale simulations: A quasi static approach. EOS Transactions, American Geophysical Union, Fall Meeting Supplement 2007; 88(52):Abstract T43E-04.

22. $\mathrm{Xu} \mathrm{W}$, Germanovich LN. Excess pore pressure resulting from methane hydrate dissociation in marine sediments: A theoretical approach. Journal of Geophysical Research 2006; 111:B01 104.

23. Yun TS, Santamarina JC, Ruppel C. Mechanical properties of sand, silt, and clay containing tetrahydrofuran hydrate. Journal of Geophysical Research 2007; 112(B04106).

24. Press WH, Flannery BP, Teukolsky SA, Vetterling WT. Numerical Recipes in Fortran 77. Cambridge University Press: New York, 1986.

25. Landau LD, Lifshitz EM. Theory of Elasticity. Course of Theoretical Physics, Vol 7, Elsevier: Burlington, MA, 1986.

26. O'Sullivan C. The application of discrete element modelling to finite deformation problems in geomechanics. PhD Thesis, University of California, Berkeley, CA 2002.

27. Bagi K. An algorithm to generate random dense arrangements for discrete element simulations of granular assemblies. Granular Matter 2005; 7(1):31-43.

28. $\mathrm{Ng}$ TT, Wang C. Comparison of a 3-D DEM simulation with MRI data. International Journal for Numerical and Analytical Methods in Geomechanics 2001; 25(5):497-507.

29. Fu Y. Experimental quantification and DEM simulation of micro-macro behaviors of granular materials using x-ray tomography imaging. PhD Thesis, University of Louisiana, Baton Rouge, LA 2005.

30. Jin G. Physics-based modeling of sedimentary rock formation and prediction of transport properties. PhD 
Thesis, University of California, Berkeley, CA 2006.

31. Christoffersen J, Mehrabadi MM, Nemat-Nasser S. A micromechanical description of granular material behavior. Journal of Applied Mechanics 1981; 48(2):339-344.

32. Bagi K. Analysis of microstructural strain tensors for granular assemblies. International Journal of Solids and Structures 2006; 43(10):3166-3184.

33. Johnson DL, Schwartz LM, Elata D, Berryman JG, Hornby B, Norris AN. Linear and nonlinear elasticity of granular media: stress-induced anisotropy of a random sphere pack. Journal of Applied Mechanics 1998; 65:380-388.

34. Makse HA, Gland N, Johnson DL, Schwartz LM. Why effective medium theory fails in granular materials. Physical Review Letters 1999; 83(24):5070-5073.

35. Domenico SN. Elastic properties of unconsolidated porous sand reservoirs. Geophysics 1977; 42(7):13391368.

36. Yong RN. Soil properties and behaviour. Developments in geotechnical engineering, Elsevier Scientific Pub. Co.: Amsterdam; New York, 1975.

37. Liou JC, Pan YW. Fabric evolution of granular assembly under KO loading/unloading. International Journal for Numerical and Analytical Methods in Geomechanics 2003; 27(13):1099-1122.

38. Yin H. Acoustic velocity and attenuation of rocks: isotropy, intrinsic anisotropy, and stress-induced anisotropy. PhD Thesis, Stanford University, Stanford, CA 1993.

39. Walton K. The effective elastic moduli of a random packing of spheres. Journal of the Mechanics and Physics of Solids 1987; 35:213-226.

40. Goddard JD. Nonlinear elasticity and pressure-dependent wave speeds in granular media. Proceedings of the Royal Society of London, Series A 1990; 430:105-131.

41. Pride SR. Relationships between seismic and hydrological properties. Hydrogeophysics, Rubin Y, Hubbard S (eds.). Springer: Dordrecht, The Netherlands, 2005.

42. Makse HA, Gland N, Johnson DL, Schwartz L. Granular packings: nonlinear elasticity, sound propagation and collective relaxation dynamics. Physical Review E 2004; 70:061302.

43. Majmudar TS, Behringer RP. Contact force measurements and stress-induced anisotropy in granular materials. Nature 2005; 435:1079-1082.

44. Durham W, Stern L, Kirby S, Circone S. Rheological comparisons and structural imaging of sI and sII endmember gas hydrates and hydrate/sediment aggregates. Proceedings of the 5th International Conference on Gas Hydrates, 26-29 September 2004, Trondheim, Norway, 2005.

45. Biot MA, Willis DG. The elastic coefficients of the theory of consolidation. Journal of Applied Mechanics 1957; 24:594-601.

46. Guerin G. Acoustic and thermal characterization of oil migration, gas hydrates formation and silica diagenesis. PhD Thesis, Columbia University, New York, NY 2000.

47. Hart DJ, Wang HF. Laboratory measurements of a complete set of poroelastic moduli for Berea sandstone and Indian limestone. Journal of Geophysical Research 1995; 100(B9):17 741-17 751.

48. Rutqvist J, Moridis GJ. Numerical studies of geomechanical stability of hydrate-bearing sediments. 2007 Offshore Technology Conference, 30 April - 3 May, Houston, TX, 2007. 